
$\$$ Research Square
Preprints are preliminary reports that have not undergone peer review.
They should not be considered conclusive, used to inform clinical practice, or referenced by the media as validated information.

\title{
The effectiveness of Information-Motivation and Behavioral skills (IMB) model of self-care in early pregnancy to prevent gestational diabetes mellitus in Iranian overweight and obese women: A Randomized Controlled Trial
}

Narges Sadat Motahari-Tabari

Mahbobeh Faramarzi

Marjan Ahmad Shirvani

Afsaneh Bakhtiari

Shabnam Omidvar

Fatemeh Nasiriamiri ( $\square$ nasiri_fa@yahoo.com )

Babol University of Medical Science https://orcid.org/0000-0001-6794-7627

Research

Keywords: Gestational Diabetes, Obesity, Overweight, Information-Motivation-Behavioral Skills Model Posted Date: February 25th, 2020

DOI: https://doi.org/10.21203/rs.2.24420/v1

License: (c) (7) This work is licensed under a Creative Commons Attribution 4.0 International License. Read Full License 


\section{Abstract}

Background: Gestational diabetes is one of the most common metabolic dysfunction in pregnancy and as overweight and obesity are of the major risk factors, this study aimed to determine the effect of Information-Motivation and Behavioral skills (IMB) model-based counseling on preventing gestational diabetes in overweight and obese pregnant women.

Methods: A randomised controlled trial (RCT) was conducted involving pregnant women who are overweight (BMl >25 to $29.9 \mathrm{k} / \mathrm{gm} 2$ ) or obese (BMl >30 k/gm2), at the 12 to 16 weeks gestation and recruited from the Prenatal Clinic of Rohani Educational Hospital in Babol medical university, Iran, women in the intervention group will receive a program informed four sessions by the Information-Motivation and Behavioral skills. The control group received routine care. Blood glucose was measured before and 8 weeks after the intervention. Descriptive and inferential statistics including mean, standard deviation, frequency, t-test, chi-square and ANCOVA were used.

Results: The prevalence of gestational diabetes was lower in the intervention group than the routine care group ( $10 \%$ and $29.9 \%$, respectively, $R R=0.33, \mathrm{Cl} 95 \%(0.15-0.74) \quad p=0.004)$ as well as mean fasting blood glucose $(d=0.28, P=0.07)$, and glucose tolerance test at the first and second hour $(d=0.41$ and $d=0.73$, respectively, $p<0.01)$.

Conclusions: Our data suggest that women that IMB model-based counseling on self-care in early pregnancy in overweight and obese pregnant women can be effective in preventing gestational diabetes.

Keywords: Gestational Diabetes, Obesity, Overweight, Information-Motivation-Behavioral Skills Model Name of the registry: Comparison of the effectiveness of counseling based on health promoting behaviors on fasting blood glucose and glucose tolerance test in pregnant and overweight and obese women

IRCT registration number: IRCT20120125008822N3

Registration date: 2018-07-05, 1397/04/14Registration timing: prospective https://en.irct.ir/trial/32150

\section{Background}

Obesity is a rapidly growing global epidemic that increases the burden of disease and decreases life expectancy [1, 2]. Iran is no exception with a prevalence of $21.7 \%$ among people over 18 [3]. Obesity contributes to increased morbidity and mortality [4]. Adverse fetal complications, metabolic diseases, preeclampsia, increased mortality and morbidity, and insulin resistance and diabetes are among adverse consequences of obesity during pregnancy [5-7].

The constant needs of a fetus for nutrients and oxygen increase insulin demand which, in turn, lead to a condition, called gestational diabetes, if there is no balance between insulin secretion and resistance [8]. 
Gestational diabetes is metabolic dysfunction, which is first developed or diagnosed during pregnancy [9]. It contributes to fetal complications, such as macrosomia, hyperinsulinemia, preterm labor, and shoulder dystocia, and maternal complications, such as hypertension, increased risk of preeclampsia and eclampsia, and difficult birth. However, gestational diabetes can cause long-term complications for mothers and infants in the future [10-13]. Therefore, gestational diabetes is of particular importance in pregnant women. As a result, various methods have been studied for preventing gestational diabetes [14-16]. Obviously, one of the most important preventive strategies is to control overweight and obesity which are closely connected to diabetes [17]. Diet change, physical activity and exercise [18], mental and social health [19], lifestyle change [20], and weight loss in a year before pregnancy [21] are among effective factors in preventing diabetes. Weight loss is one of the most important factors in preventing gestational diabetes [22]. On the other hand, strengthening motivation is an important factor in weight loss by encouraging people to continue their weight loss program [23].

IMB is a comprehensive model for promoting health behaviors, especially in chronic diseases [24]. This model has proved successful in explaining health behaviors over the past ten years [16]. Numerous studies have demonstrated the positive effect of IMB model-based interventions on improving self-care behaviors and related health outcomes in patients with diabetes through weight loss and $\mathrm{HbA} 1 \mathrm{C}$ reduction $[24,25]$.

Considering the background of effective studies on IMB model, the importance of gestational diabetes and its impact on maternal and fetal health, and the increased prevalence of overweight and obesity, some methods are needed to reduce the likelihood of obesity in order to reduce the prevalence of gestational diabetes. Therefore, considering that the IMB model is an easy, inexpensive, accessible and sustainable method, the aim of this study was to measure the effectiveness of early intensive lifestyle intervention by the IMB model in preventing the incidence of gestational diabetes in overweight and obese pregnant women. The hypothesis was that women in the intervention group had fewer diagnoses of GDM than women in the usual care group on the incidence of GDM in overweight and obese women.

\section{Materials And Methods}

This randomized controlled trial was approved by the Ethics Committee of Babol University of Medical Sciences, in 2018, and registered at the Iranian Registry of Clinical Trials (IRCT20120125008822N3). The study population consisted of all pregnant women at the end of their first trimester of pregnancy who visited the Prenatal Clinic of Rohani Educational Hospital in Babol, Iran. The study will be conducted and reported using the CONSORT recommendations [26].

\subsection{Sample size estimation}

The Results of the study by Quinlivan et al. were used to determine the sample size based on the prevalence of gestational diabetes in the intervention and the usual care groups [27]. In this study, the overweight women $(\mathrm{BMI}>25)$ received educational intervention about diet, increased physical activity, 
and stress management. The incidence of gestational diabetes mellitus in the 24-28 weeks of gestation was $29 \%$ in the control group and $6 \%$ in the intervention group. The sample size was estimated at 110 (55 participants for each group) at a confidence level of $95 \%$, test power of $80 \%$, a two-tailed test, and using the formula of comparison between two ratios in G-power. Due to the chance of $20 \%$ drop out, the sample size for each group increased to 74. Finally, the total sample size was 148 .

\subsection{Randomization procedure}

A computer generated randomization sequence will be used to facilitate the permuted block randomization of participants. Random allocation to the intervention or the usual care group will be conducted by an individual who is independent to the study by drawing opaque numbered envelopes consecutively, to reveal the group allocation.

\subsection{Participants}

Inclusion criteria were pregnant women over 16 years old, a BMI of $\geq 25 \mathrm{Kg} / \mathrm{m}^{2}$, basic literacy, singleton pregnancy, normal results from the first-trimester test for fasting glucose based on the national and international protocols (maximally, $92 \mathrm{mg} / \mathrm{dl}[28,29]$ and gestational age between 12 weeks and 1 day to 16 weeks and 6 days. Exclusion criteria will include women: with pre-existing diabetes (types 1 and 2), a past history of gestational diabetes, unable to give informed consent in Persian, currently experiencing vaginal bleeding or with severe medical conditions preventing them from being able to undertake regular low impact exercise. Also they were withdrawal from the study at each stage and incomplete questionnaire completion.

Eligible participants were first selected from the medical records of pregnant women who visited the Prenatal Care Clinic. They were contacted and invited to attend the clinic. Participants were susceptible to diabetes due to high BMI. After a thorough explanation of the aims of the study and obtaining written and oral informed consent, the participants were randomly assigned to counseling and the usual care groups. Before the study, all participants completed the demographic questionnaire and the results of the initial blood glucose test were recorded from their health records.

\subsection{Intervention}

Education and counseling included four sessions based on the IMB model. The first counseling session lasted for 90 minutes on the first day of the visit. Subsequent 60-90-minute sessions were held in person (three sessions) each week. Counseling was provided by the researcher using the IMB model during a four-week period according to the timetable agreed on with the women in the maternal education room of the clinic. In addition, the participants were provided with a booklet on how to perform appropriate physical activities during pregnancy. During each session, PowerPoint presentations, image presentation and question and answer techniques were used. First session: counseling for obesity, nutrition during pregnancy and the impact of obesity on gestational diabetes; second session: counseling on how to do physical activities during pregnancy and its benefits; third session: counseling for diabetes self-care, how to manage stress in pregnancy and its effect on gestational diabetes; fourth session: counseling on the 
impact of motivation on mental health and continuity of chronic disease control. Standard maternal care was also provided to both groups. Women in the control group will receive usual care. Usual care in this setting involves initial weighing to calculate BMI [30]. At the end of the counseling sessions, mean plasma glucose levels were measured for both groups at 24 to 28 weeks of gestation. A two-hour, 75gram oral glucose tolerance test (OGTT) was used to measure blood glucose (fasting glucose, and one hour and two hours later). Whenever one blood glucose test result was higher than or equal to normal index $(92,180$ and $153 \mathrm{mg} / \mathrm{dl}$, respectively) $[28,29]$ diagnosis of gestational diabetes was made and the pregnant woman was referred to a gynecologist.

\subsection{Blinding}

All participating women will provide written consent prior to randomization however women will not be blinded to allocation. Due to the type of intervention, blinding of the researchers to allocation is also not realistic. Analysis will be performed by a person independent to the research team to avoid assessment bias.

\subsection{Data Collection Instruments}

The data collection instrument was a two-part questionnaire including demographic characteristics and para-clinical tests reports, which was completed by inquiring the participants and from their medical records by the author. The first part of the questionnaire included demographic, social, reproductive, and laboratory information with 17 questions about weight, height, age, number of pregnancies, date of the last menstruation, expected date of delivery, gestational age by week, etc. The second part of the questionnaire consisted of baseline FBS and the result of re-measuring FBS and OGTT at weeks 24-28 with $75 \mathrm{~g}$ glucose according to ADA criteria, the national protocol and the World Health Organization's protocol [28-29-31]. It should be noted that fasting glucose test and glucose tolerance test were both performed at the same center.

\subsection{Data Analysis}

The collected data was inputted into SPSS 21 with an appropriate code. Demographic variables were reported using descriptive statistics (mean and standard deviation for quantitative variables and frequency for qualitative variables) and the between-group comparison was one using t-test and chisquare. An ANCOVA was used to compare blood glucose between groups. Leven test was used to test homogeneity of variance and homogeneity of regression coefficients. In addition, the results of the glucose tolerance test were compared between the two groups by independent $t$-test. To compare the incidence of diabetes between the two groups, chi-square and relative risk calculations were used.

\section{Results}

Of 162 pregnant women evaluated, 148 met the inclusion criteria and agreed to participate in this study. Others were excluded because of type 2 diabetes, high blood pressure, and thyroid disorders. Finally, 137 
participants were divided into two groups (70 participants in the counseling and 67 participants in the routine care group) (Flowchart 1 ).

Comparison of socio-demographic data showed that there was no significant difference between the two groups (Table 1). 
Table 1

Comparison of demographic and social characteristics of the samples in two groups of counseling based on motivational model and behavioral and control skills

\begin{tabular}{|c|c|c|}
\hline Variable & $\begin{array}{l}\text { Intervention group } \\
(\mathrm{N}=70) \\
\text { Number (Percent) }\end{array}$ & $\begin{array}{l}\text { Routine care group } \\
(\mathrm{N}=67) \\
\text { Number (Percent) }\end{array}$ \\
\hline Age mother (year) ${ }^{*}$ & 29.04(5.94) & $27.68(5.72)$ \\
\hline BMI $\left(\mathrm{Kg} / \mathrm{m}^{2}\right)$ & $32.44(3.45)$ & $33.20(4.60)$ \\
\hline \multicolumn{3}{|l|}{ Education } \\
\hline Under diploma & 11(15.7) & 13(19.4) \\
\hline Diploma & $39(55.7)$ & $31(46.3)$ \\
\hline More than diploma & $20(28.6)$ & 23(34.3) \\
\hline \multicolumn{3}{|l|}{ Job mother } \\
\hline Housewife & 61(87.1) & $59(88.1)$ \\
\hline Employee & $9(12.9)$ & $8(11.9)$ \\
\hline \multicolumn{3}{|l|}{ Job father } \\
\hline Employee & 11(15.7) & 14(20.9) \\
\hline Worker & 12(17.1) & $6(9)$ \\
\hline Self-employment & $47(67.1)$ & $47(70.1)$ \\
\hline \multicolumn{3}{|l|}{ Income } \\
\hline Enough & $26(37.1)$ & 18(26.9) \\
\hline Enough to be enough & $40(57.1)$ & $44(65.7)$ \\
\hline Not enouph & $5(7.5)$ & $4(5.7)$ \\
\hline \multicolumn{3}{|l|}{ Location } \\
\hline City & $48(68.6)$ & $37(55.2)$ \\
\hline Rural & $22(31.4)$ & $30(44.8)$ \\
\hline \multicolumn{3}{|l|}{ Gravity } \\
\hline Noli Par & $28(40)$ & $33(49.01)$ \\
\hline Molti par & $42(60)$ & $34(50.7)$ \\
\hline
\end{tabular}




\begin{tabular}{|lll|}
\hline Variable & $\begin{array}{l}\text { Intervention group } \\
(\mathbf{N}=70) \\
\text { Number (Percent) }\end{array}$ & $\begin{array}{l}\text { Routine care group } \\
(\mathbf{N}=67) \\
\text { Number (Percent) }\end{array}$ \\
\hline Type of delivery & $28(40)$ & $33(49.3)$ \\
\hline No delivery & $15(21.4)$ & $13(86.7)$ \\
\hline NVD & $27(38.6)$ & $21(31.3)$ \\
\hline C/S & $0(0)$ & $1(1.5)$ \\
\hline Anomaly history & $4(5.7)$ & $1(1.5)$ \\
\hline Macrosomal history & $2(2.9)$ & $2(3)$ \\
\hline History of stillbirth & $2(2.9)$ & $1(1.5)$ \\
\hline History of preterm labor & & \\
\hline Variables were reported as numbers $(\%)$, except for cases * reported as mean (Standard Deviation) & \\
\hline
\end{tabular}

\subsection{Fasting blood glucose}

Second-trimester fasting blood glucose was lower in both groups than the first trimester, but the mean difference in the two groups increased in weeks $24-28$, with fasting blood glucose level being significantly lower in the counseling group than the routine care group (Table 2).

Table 2

Comparison of fasting blood sugar levels in routine care and Intervention groups

\begin{tabular}{|c|c|c|c|c|c|c|c|}
\hline \multirow[t]{2}{*}{ Variables } & \multicolumn{2}{|c|}{$\begin{array}{l}\text { Intervention group } \\
(\mathrm{N}=70) \\
\text { Mean(SD) }\end{array}$} & \multicolumn{2}{|c|}{$\begin{array}{l}\text { Routine care group } \\
(\mathrm{N}=67) \\
\text { Mean(SD) }\end{array}$} & \multirow[t]{2}{*}{$F$} & \multirow[t]{2}{*}{$\begin{array}{l}\text { Effect size } \\
\text { (Cohen's d) }\end{array}$} & \multirow[t]{2}{*}{$P$ value } \\
\hline & After & Before & After & Before & & & \\
\hline FBS & $\begin{array}{l}80.50 \\
(8.21)\end{array}$ & $\begin{array}{l}83.54 \\
(6.43)\end{array}$ & $\begin{array}{l}83.80 \\
(10.72)\end{array}$ & $\begin{array}{l}84.86 \\
(7.69)\end{array}$ & 3.13 & 0.28 & 0.07 \\
\hline
\end{tabular}

Comparison between groups using ANCOVA demonstrated that the difference in fasting blood glucose between the two groups was close to significant level, though it was not statistically significant. The mean difference between the two groups was $d=0.28$, indicating a slight difference (Table 2 ).

\subsection{OGGT test}

According to the independent t-test, the mean blood glucose in the first-hour and second-hour glucose tolerance tests was significantly lower in the counseling group. In addition, the mean difference of blood 
glucose level in the first-hour screening of the glucose tolerance test was $d=0.41$ and in the second hour of the glucose tolerance test was $d=0.73$, which was significantly lower in the counseling group than the control group (Table 3).

Table 3

Comparison of first and second hour glucose tolerance test in routine care and Intervention groups

\begin{tabular}{|lllll|}
\hline Glucose tolerance test & $\begin{array}{l}\text { Intervention group }(\mathbf{N}=70) \\
\text { Mean(SD) }\end{array}$ & $\begin{array}{l}\text { routine care group } \\
\mathbf{N}=67) \\
\text { Mean(SD) }\end{array}$ & $\begin{array}{l}\text { Effect size } \\
\text { (Cohen's d) }\end{array}$ & P value \\
\hline $1 \mathrm{~h}$ & $\begin{array}{l}126.97 \\
(22.48)\end{array}$ & $\begin{array}{l}137.62 \\
(28.07)\end{array}$ & 0.41 & $0.01>$ \\
\hline $2 \mathrm{~h}$ & $100.25(20.99)$ & $\begin{array}{l}117.05 \\
(24.31)\end{array}$ & 0.73 & $0.01>$ \\
\hline
\end{tabular}

\subsection{Incidence of gestational diabetes}

In the follow-up, $80.3 \%$ participants did not have gestational diabetes and $19.7 \%$ had gestational diabetes. Chi-square test showed that there was a significant difference between the two groups in the incidence of diabetes $(P=0.004)$. The relative risk of gestational diabetes in overweight and obese women with IMB counseling reduced by $67 \%$, and the relative risk of diabetes in the intervention group was $0.33,95 \% \mathrm{Cl} 95 \%=0.15-0.74$ (Table 4 ).

Table 4

Comparison of diabetes frequency (according to national protocol) between counseling and routine care groups

\begin{tabular}{|c|c|c|c|c|c|c|}
\hline groups & $\begin{array}{l}\text { Intervention } \\
\text { group } \\
\text { Number } \\
\text { (Percent) }\end{array}$ & $\begin{array}{l}\text { routine care } \\
\text { group } \\
\text { Number } \\
\text { (Percent) }\end{array}$ & $\begin{array}{l}\text { Total } \\
\text { Number } \\
\text { (Percent) }\end{array}$ & $\begin{array}{l}\text { Chi- } \\
\text { square }\end{array}$ & DF & $\begin{array}{l}\mathrm{P} \text { - } \\
\text { value }\end{array}$ \\
\hline $\begin{array}{l}\text { No } \\
\text { diabetes }\end{array}$ & $\begin{array}{l}63 \\
(90.0)\end{array}$ & $\begin{array}{l}47 \\
(70.1)\end{array}$ & $\begin{array}{l}110 \\
(80.3)\end{array}$ & 8.525 & 1 & 0.004 \\
\hline Diabetic & $\begin{array}{l}7 \\
(10.0)\end{array}$ & $\begin{array}{l}20 \\
(29.9)\end{array}$ & $\begin{array}{l}27 \\
(19.7)\end{array}$ & & & \\
\hline Total & $\begin{array}{l}70 \\
(51.1)\end{array}$ & $\begin{array}{l}67 \\
(48.9)\end{array}$ & $\begin{array}{l}137 \\
(100.0)\end{array}$ & & & \\
\hline
\end{tabular}

\section{Discussion}

Results of the comparison of mean fasting glucose in overweight and obese pregnant women in both counseling and routine care groups showed that the variance of blood glucose was similar between 
groups. Intergroup comparison suggested that there was a difference in fasting blood glucose between the two groups, which was not statistically significant. In addition, the prevalence of gestational diabetes was significantly lower in the counseling group.

A study by Quinlivan et al., which examined the four-step approach of motivational counseling in prenatal care in overweight and obese women, found that a significant reduction in the incidence of gestational diabetes occurs with the application of this protocol [27] which is consistent with the results of the present study. In a study, Luoto et al. investigated the effect of the IMB model on self-care in people with type 2 diabetes. During this study, it was observed that using this model significantly controls glycemia [32], which is consistent with the present study. In our study, using this model also reduced glycemia but this reduction was not significant. In a study which aimed to evaluate the effect of IMB model on adherence to recommended treatment regimens in patients with diabetes in Babol, using this model was effective on improving self-care and quality of life of patients with diabetes [33]. In addition, in the study by Khosravi et al., which aimed to investigate the effect of IMB model on improving body weight control and area of the limbs in women undergoing weight loss surgery in Shiraz, the results indicated that this model has a significant effect on weight control and area of the limbs in women undergoing weight loss surgery [34], which relatively complies with the present study. In these studies, the effect of the model on diabetes improvement and weight control was significant. In our study, although there was a difference in fasting blood glucose between the two groups, this difference was not statistically significant. This may be due to the difference in the study population because the participants in the present study were pregnant women and due to pregnancy conditions, diet restriction and weight control were more stringent than other populations. In a study, the authors suggested that the IMB model may be one of the most important elements in enhancing physical activity, and fruit and vegetable consumption in adolescents. IMB could decrease HbA1c levels, weight and blood pressure over the past year [35] which complied with the present study.

The IMB model is a comprehensive model for promoting health behaviors by encouraging to improve lifestyle, reduce and maintain weight in an ideal or near-ideal state, and strengthen the will to improve health behaviors and preventive factors for chronic diseases, including blood glucose control. The most important strength of this study was the evaluation of the effects of behavioral interventions on biological variables (fasting glucose assay and glucose tolerance test). Other strengths of this study included observing the full principles of counseling, random allocation of participants, and the use of standard questionnaires.

We recognized that there are limitations in our study. First, this research was restricted to the pregnant women at a single hospital. Thus, the results cannot be generalized to all overweight and obese pregnant women. it is suggested that subsequent studies be carried out with a larger number of samples and multicenter. Second, this study might be the lack of blindness due to the nature of the intervention.Third, clinical information including pre-pregnancy height and weight were self-reported, which tend to be slightly different from when directly measured; however, these approaches had been used in the literature [36].

Page $10 / 16$ 


\section{Conclusion}

Although A lifestyle intervention in early pregnancy by IMB counseling in overweight and obese pregnant women could not significantly decrease fasting blood glucose, there was a significant difference in mean glucose in the two groups; in addition, we concluded that the lifestyle intervention is clinically efficacious, which has decreased the incidence of gestational diabetes in the intervention group, so it could predict the positive effect of this model on gestational diabetes prevention. It is recommended to investigate the effect of IMB counseling on other maternal and fetal outcomes in overweight and obese pregnant women.

\section{Declarations}

\section{Ethics approval and consent to participate}

All procedures were carried out on the basis of ethics standards of the responsible committee on human experimentation (institutional and national) and to the Declaration of Helsinki. In addition, the ethics Committee of BUMS approved the study (NO. IR.MUBABOL.HRI.REC.1397.068). All participants provided an informed written and signed consent form. All patients provided written informed consent.

\section{Consent for publication}

We confirm that the manuscript entitled "The effectiveness of Information-Motivation and Behavioral skills (IMB) model on self-care in early pregnancy to prevent gestational diabetes mellitus in Iranian overweight and obese women: A randomized controlled trial" has been read and approved by all named authors and that there are no other persons who satisfied the criteria for authorship but are not listed. We further confirm that the order of authors listed in the manuscript has been approved by all of us.

The manuscript submitted for publication in Diabetology\& Metabolic Syndrome has been read and approved by all authors, has not been published, totally or partly, in any other journal even in Persian language and if accepted for publication in this Journal, it will not be published in any other periodicals. Besides, it is confirmed that this article has not been submitted simultaneously to other journals in Iran and/or other countries. I accept the responsibility of the scientific integrity of the manuscript contents.

We confirm that we have given due consideration to the protection of intellectual property associated with this work and that there are no impediments to publication, including the timing of publication, with respect to intellectual property. In so doing we confirm that we have followed the regulations of our institutions concerning intellectual property.

We understand that the Corresponding Author is the sole contact for the Editorial process (including Editorial Manager and direct communications with the office). She is responsible for communicating with the other authors about progress, submissions of revisions and final approval of proofs. 
Signed by ALL AUTHORS as follows: (add more pages as needed)

\begin{tabular}{|l|l|l|}
\hline Author name & Signature & Date \\
\hline Narges Sadat Motahari-Tabari & 16 February 2020 \\
\hline Mahbobeh Faramarzi & & 16 February 2020 \\
\hline Marjan Ahmad Shirvani & & 16 February 2020 \\
\hline Fatemeh Nasiri-Amiri & & 16 February 2020 \\
\hline Shabnam Omidvar & & 16 \\
\hline
\end{tabular}

Availability of data and material

We have had access to all the data in the study (for original research articles) and accept responsibility for its validity.

Competing interests

No potential conflicts of interest relevant to this article were reported.

\section{Funding}

This study was approved and funded by the Deputy for Research of Babol University of Medical

Sciences (Grant No: 9705313).

\section{Authors' contributions}

Motahari-Tabari N. conceived and designed the study, she also did data collection. Faramarzi M, Shirvani M, Bakhtiari A, Omidvar S, Bakouei F reviewed the literature, analyzed and interpreted data, drafted and prepared the manuscript. Nasiri-Amiri F developed the first draft of this manuscript and had full access to 
all the data in the study and takes responsibility for the integrity of the data and the accuracy of the data analysis. All authors approved the final version of manuscript.

\section{Acknowledgements}

This paper was a thesis for receiving M.Sc. degree in counseling in Midwifery which approved by Babol University of Medical Sciences. The authors would like to thank this university for its financial support. We also would like to extend our sincere thanks to all the participants who helped us in this research despite their difficult pregnancy conditions.

\section{References}

1. Kramer H, Kalantar-Zadeh K. Obesity, preterm birth and kidney disease: a global epidemic. Nephrology Dialysis Transplantation. 2018.

2. Abarca-Gómez L, Abdeen ZA, Hamid ZA, Abu-Rmeileh NM, Acosta-Cazares B, Acuin C, et al. Worldwide trends in body-mass index, underweight, overweight, and obesity from 1975 to 2016: a pooled analysis of 2416 population-based measurement studies in 128.9 million children, adolescents, and adults. The Lancet. 2017;390(10113):2627-42.

3. Rahmani A, Sayehmiri K, Asadollahi K, Sarokhani D, Islami F, Sarokhani M. Investigation of the prevalence of obesity in Iran: a systematic review and meta-analysis study. Acta Medica Iranica. 2015:596-607.

4. Johnsson IW, Haglund B, Ahlsson F, Gustafsson J. A high birth weight is associated with increased risk of type 2 diabetes and obesity. Pediatric obesity. 2015;10(2):77-83.

5. Ramsey PS, Schenken RS, Pi-Sunyer FX. Obesity in pregnancy: Complications and maternal management. UpToDate®[CJ Lockwood, F Pi-Sunyer and V Barss, editors] https://www uptodate com/contents/obesity-inpregnancy-complications-and-maternal-management. 2017:3-150.

6. Palomba S, De Wilde MA, Falbo A, Koster MP, La Sala GB, Fauser BC. Pregnancy complications in women with polycystic ovary syndrome. Human reproduction update. 2015;21(5):575-92.

7. Feresu SA, Wang Y, Dickinson S. Relationship between maternal obesity and prenatal, metabolic syndrome, obstetrical and perinatal complications of pregnancy in Indiana, 2008-2010. BMC pregnancy and childbirth. 2015;15(1):266.

8. Mottola MF, Artal R. Role of exercise in reducing gestational diabetes mellitus. Clinical obstetrics and gynecology. 2016;59(3):620-8.

9. Buchanan TA, Xiang AH, Page KA. Gestational diabetes mellitus: risks and management during and after pregnancy. Nature Reviews Endocrinology. 2012;8(11):639.

10. Sha T, Yin X, Cheng W, Massey IY. Pregnancy-related complications and perinatal outcomes resulting from transfer of cryopreserved versus fresh embryos in vitro fertilization: a meta-analysis. Fertility and sterility. 2018;109(2):330-42. e9. 
11. Ovesen PG, Jensen DM, Damm P, Rasmussen S, Kesmodel US. Maternal and neonatal outcomes in pregnancies complicated by gestational diabetes. A nation-wide study. The Journal of Maternal-Fetal \& Neonatal Medicine. 2015;28(14):1720-4.

12. Kampmann U, Madsen LR, Skajaa GO, Iversen DS, Moeller N, Ovesen P. Gestational diabetes: a clinical update. World journal of diabetes. 2015;6(8):1065.

13. Kumari A, Singh C. A Review on Gestational Diabetes Mellitus during Pregnancy. Journal of Drug Delivery and Therapeutics. 2019;9(3-s):1123-5.

14. Madhuvrata P, Govinden G, Bustani R, Song S, Farrell T. Prevention of gestational diabetes in pregnant women with risk factors for gestational diabetes: a systematic review and meta-analysis of randomised trials. Obstetric medicine. 2015;8(2):68-85.

15. Koivusalo SB, Rönö K, Klemetti MM, Roine RP, Lindström J, Erkkola M, et al. Gestational diabetes mellitus can be prevented by lifestyle intervention: the Finnish Gestational Diabetes Prevention Study (RADIEL): a randomized controlled trial. Diabetes care. 2016;39(1):24-30.

16. Bain E, Crane M, Tieu J, Han S, Crowther CA, Middleton P. Diet and exercise interventions for preventing gestational diabetes mellitus. Cochrane database of systematic reviews. 2015(4).

17. Wang $C$, Wei $Y$, Zhang $X$, Zhang $Y, X u$ Q, Sun $Y$, et al. A randomized clinical trial of exercise during pregnancy to prevent gestational diabetes mellitus and improve pregnancy outcome in overweight and obese pregnant women. American journal of obstetrics and gynecology. 2017;216(4):340-51.

18. Gilbert L, Gross J, Lanzi S, Quansah DY, Puder J, Horsch A. How diet, physical activity and psychosocial well-being interact in women with gestational diabetes mellitus: an integrative review. BMC pregnancy and childbirth. 2019;19(1):60.

19. Rönö K, Stach-Lempinen B, Eriksson JG, Pöyhönen-Alho M, Klemetti MM, Roine RP, et al. Prevention of gestational diabetes with a prepregnancy lifestyle intervention-findings from a randomized controlled trial. International Journal of Women's Health. 2018;10:493.

20. Liu H, Wang L, Zhang S, Leng J, Li N, Li W, et al. One-year weight losses in the Tianjin Gestational Diabetes Mellitus Prevention Programme: A randomized clinical trial. Diabetes, obesity and metabolism. 2018;20(5):1246-55.

21. LEBLANC ES, SMITH N, VESCO K, ROSALES AG, STEVENS VJ. 195-LB: How Does a Prepregnancy Weight Loss Program Affect Gestational Diabetes Risk? : Am Diabetes Assoc; 2019.

22. Tigay JH, Thompson M, Sutton D, Lesley M. Motivation for weight loss. The Journal for Nurse Practitioners. 2016;12(4):e169-e73.

23. Osborn CY, Rivet Amico K, Fisher WA, Egede LE, Fisher JD. An information-motivation-behavioral skills analysis of diet and exercise behavior in Puerto Ricans with diabetes. Journal of health psychology. 2010;15(8):1201-13.

24. Gao J, Wang J, Zhu Y, Yu J. Validation of an information-motivation-behavioral skills model of selfcare among Chinese adults with type 2 diabetes. BMC Public Health. 2013;13(1):100.

25. Osborn CY, Egede LE. Validation of an Information-Motivation-Behavioral Skills model of diabetes self-care (IMB-DSC). Patient education and counseling. 2010;79(1):49-54. 
26. Moher D, Schulz KF, Altman DG, Consort: The CONSORT statement: revised recommendations for improving the quality of reports of parallel group randomized trials. BMC Med Res Methodol 2001, 1:2[Research Support, Non-U.S. Gov't].

27. Quinlivan JA, Lam LT, Fisher J. A randomised trial of a four-step multidisciplinary approach to the antenatal care of obese pregnant women. Australian and New Zealand Journal of Obstetrics and Gynaecology. 2011;51(2):141-6.

28. WHO recommendation on the diagnosis of gestational diabetes in pregnancy Geneva: World Health Organization; 08 March 2018 [Available from: https://extranet.who.int/rhl/topics/preconceptionpregnancy-childbirth-and-postpartum-care/antenatal-care/who-recommendation-diagnosisgestational-diabetes-pregnancy- 0 .

29. The Ministry of Health and Medical Education OoFHaP. In: Education TMoHaM, editor. National Program maternal immune. IRAN: Department of Maternal Health; 2016

30. Maternity and Newborn Clinical Network: Care of the obese pregnant woman and Weight Management in Pregnancy. Melbourne, Victoria,Australia 2011.

31. Inzucchi SE, Bergenstal RM, Buse JB, Diamant M, Ferrannini E, Nauck M, et al. Management of hyperglycemia in type 2 diabetes: a patient-centered approach: position statement of the American Diabetes Association (ADA) and the European Association for the Study of Diabetes (EASD). Diabetes Spectrum. 2012;25(3):154-71.

32. Liu T, Wu D, Wang J, Li C, Yang R, Ge S, et al. Testing the information-motivation-behavioural skills model of diabetes self-management among Chinese adults with type 2 diabetes: a protocol of a 3month follow-up study. BMJ open. 2018;8(10):e020894.

33. AkbarnatajK1 H, DoustiYA3, FakhriMK4, Shirafkan AM. Effectiveness of information-motivation and behavioral skills (IMB) model on adherence of Recommended treatment regimens in patients with type 2 diabetes. nursing development in health. 2015;6(1):27-37.

34. Khosravi S, Amini M, Poursharifi H, Sobhani Z, Sadeghian L. The Effectiveness of InformationMotivation-Behavioral Model on Improving the Weight and Body Size Among Women Undergoing Bariatric Surgery. ISMJ. 2018;21(1):81-91.

35. Fisher WA, Fisher JD, Shuper PA. Social psychology and the fight against AIDS: An informationMotivation-Behavioral skills model for the prediction and promotion of health behavior change. Advances in experimental social psychology. 50: Elsevier; 2014. p. 105-93.

36. Sun $Y$, Zhao $H$. The effectiveness of lifestyle intervention in early pregnancy to prevent gestational diabetes mellitus in Chinese overweight and obese women: A quasi-experimental study. Applied Nursing Research. Appl Nurs Res. 2016 May;30:125-30.

\section{Figures}




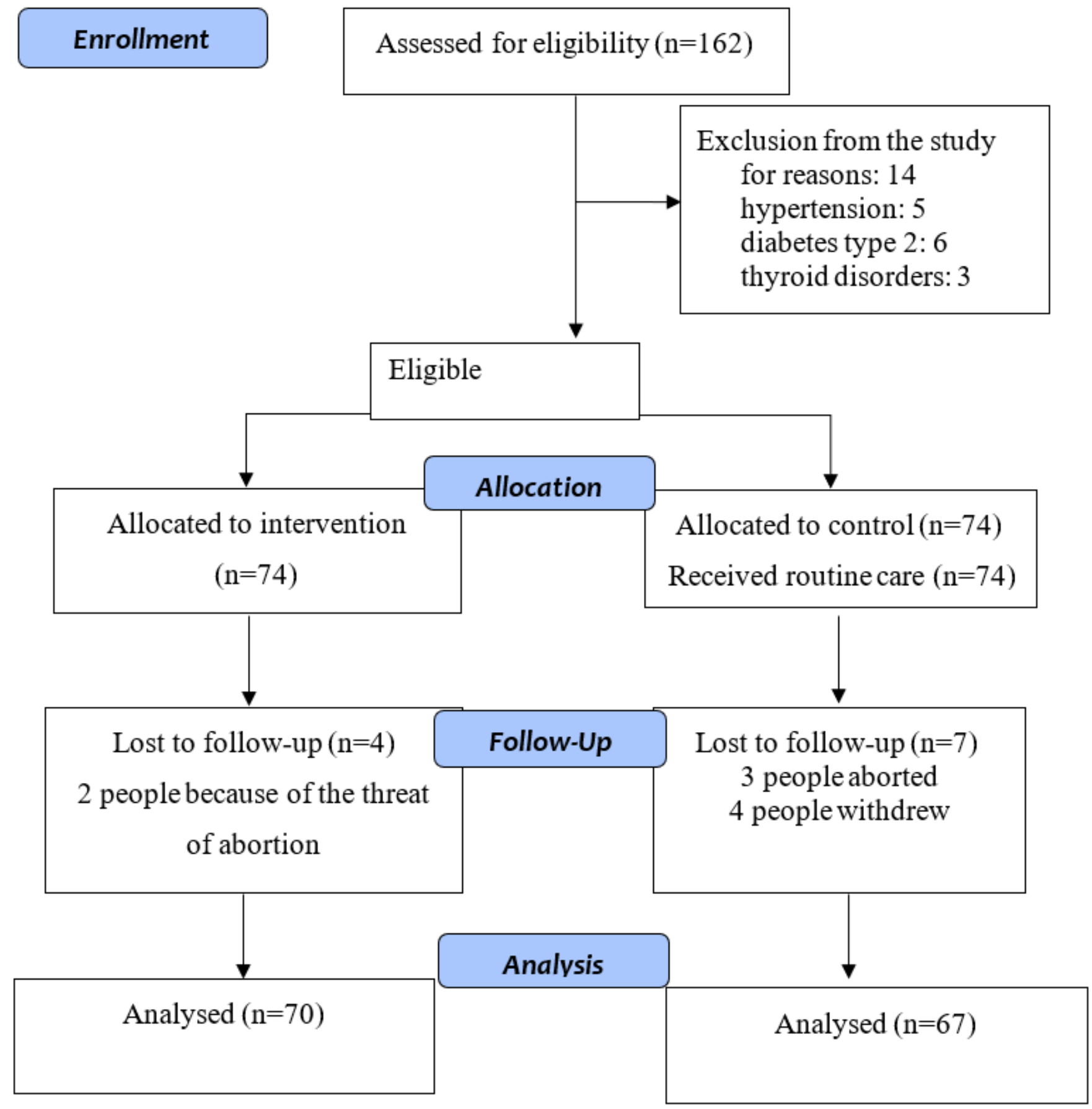

Figure 1

Process of enrollment of participants in the study 